\title{
THE ARTISTIC PLAY OF SPATIAL ORGANIZATION: SPATIAL ATTRIBUTES, SCENE ANALYSIS AND AUDITORY SPATIAL SCHEMATA
}

\author{
Gary S. Kendall \\ Northwestern University \\ Music Technology Program \\ Evanston, IL 60208 USA \\ g-kendall@northwestern.edu
}

\begin{abstract}
Electroacoustic music lacks a definitive vocabulary for describing its spatiality. Not only does it lack a vocabulary for describing the spatial attributes of individual sound sources, it lacks a vocabulary for describing how these attributes participate in artistic design and expression. Following work by Rumsey [15] the definition of spatial attributes is examined in the broader context of auditory scene analysis. A limited number of spatial attributes are found to be adequate to characterize the individual levels of organization nested within the auditory scene (levels that for acoustic music Rumsey labels as source, ensemble, room and scene). These levels are then viewed as products of both tangible spatial relationships and auditory spatial schemata, the recurrent patterns by which listeners understand the behavior of sound in space. In electroacoustic music the interrelationship of spatial attributes and spatial schemata is often engaged in a play of perceptual grouping that blurs and confounds distinctions like source and ensemble. Our ability to describe and categorize these complex interactions depends on having clear concepts and terminology so that we can recognize the crisscrossing of boundaries and the violation of conventions in this artistic interplay.
\end{abstract}

\section{INTRODUCTION}

The expanded range of its spatial palette is one of the important features that distinguish electroacoustic music from acoustic music. The situation for spatiality in electroacoustic music is similar to that for sound synthesis in the sense that the experience of the natural world provides an inspiration for creativity and research. At the same time technology enables new possibilities that reach beyond the bounds of everyday experience. Electroacoustic music's capacity to manipulate audio signals creates a context in which there can be uniquely complex interactions between spatial hearing and other domains of perception and cognition. This is especially true when electroacoustic composers play with the fundamentals of spatial organization in music by manipulating perceptual grouping and violating spatial schemata. Spatial audio, and especially spatial audio for electroacoustic music, is an artistic domain that often throws the spatial conventions of the natural world into relief by distorting or violating them. In order to appreciate the crisscrossing of boundaries and conventions in this artistic interplay, our concepts and vocabulary should be in good alignment with the listener's perceptual and cognitive processes.

\section{TERMINOLOGY}

\subsection{The Problem of Terminology}

While the spatiality of acoustic music, even 20th century acoustic music, can be discussed in commonly understood terms [5], the spatiality of electroacoustic music still lacks a definitive vocabulary. When music is performed by acoustic instruments in an acoustic environment, the physical level of description by itself often provides a workable roadmap to both the listener's experience and the composer's intent. We have a wealth of shared experiences and traditions of acoustic performance despite the perceptual complexity and individuality of many performances. In electroacoustic music, the acoustic experience has often been a reference point, but the technology of electronic reproduction expands the scope and complexity of spatiality in a radical way. Even though the apparatus may be located within a physical space and even though our spatial hearing has developed within a physical world, electronic reproduction creates the potential for an art of spatiality. Consider how the experience of a diffuse granular cloud [21] emerges from the details of the granular synthesis or how the experience of spectral bands distributed in space challenges [20] our notion of what constitutes a 'source.' Electroacoustic music hardly has the vocabulary to describe the scope of spatial possibilities or to explain the relationship of signal processing techniques to the listener's perceptions.

Recent perceptual research can help us begin to clarify our vocabulary. A great deal of relevant research has emerged from the study of spatial impression associated with subjective acoustics of concert halls [2]. In the subjective acoustics of electronic reproduction, Zacharov and Koivuniemi [22] and Rumsey [16] provide in-depth discussions and classification of perceptual spatial attributes. One particularly important area of focus has been the interrelated study of apparent source width and listener envelopment $[1,4]$. These perceptual qualities have been studied almost exclusively in the context of concert hall acoustics and more recently in the context of sound reproduction, especially in surround sound systems. And, while there is much that can be gleaned from this research, the difference between the contexts considered in the aforementioned research and the context for electroacoustic music is profound. For one thing, concert hall acoustics and reproduced sound are often partitioned conceptually into direct sound, indirect reflections and reverberation. This is reflected in a terminology that can be parsed into terms relating to the source and those relating to the environment. 
Importantly, electroacoustic music is not limited to the source-environment model. Then too, there are profound differences in the sound material. This is especially important for two reasons: spatial percepts are shaped in part by the content of the sound sources $[10,11]$ and electroacoustic composers are not limited to pre-existent acoustic sources. Not only do electroacoustic composers have the freedom to design sounds that specifically support spatial effects, but they can also explore acoustic realms that break down ecological validity!

\subsection{Spatial Attributes and Scene Analysis}

In a particularly useful discussion, Rumsey [15] considers spatial sound attributes within the framework of auditory scene analysis [3]. He states that spatial attributes "should be unambiguous and preferably unidimensional (in other words, they should represent a single perceptual construct)." Spatial attributes have to do with the tangibly three-dimensional aspects of sound including such properties as width and distance. Most importantly Rumsey ${ }^{1}$ separates spatial attributes from attributes of spaces, that is, from the properties of the rooms (or other environments). The attributes of spaces have traditionally included properties such as reverberance and liveness. The confluence of these categories had been unexamined in the subjective evaluation of concert halls and audio reproduction systems where sound sources are assumed to be contained within environments, and, in fact, Rumsey's discussion is itself directed toward such typical audio reproduction settings. This confluence of categories is essentially a misalignment of physical and perceptual acoustics. So, for example, if we shift our orientation completely to the side of the listener's auditory organization and observe that the indirect sound of early reflections influences the perception of the auditory source, then that indirect sound is essentially part of the auditory source's signal just as much as the direct sound. And, from this point on we must be particularly clear about the separation between the acoustic source signal (with all of its acoustic constituents) and the source's perceived image (with all of its perceptual spatial attributes). The separation of the categories is particularly useful in electroacoustic music where the source-in-environment model is only one of many possible spatial treatments.

Having achieved a clear separation of spatial attributes from other properties of sound in space, Rumsey goes on to examine spatial attributes within the context of auditory scene analysis. In creating an organizational framework for evaluating the kind of sound reproduction that interests him, he proposes four levels of organization that are common in the experience of listening to recorded music: source, ensemble, room and scene. These labels are meant to be more general categories of nested organization than types of acoustic sources. How these four levels of organization interact with the spatial attribute of width is illustrated in Figure 1. At the lowest level of organization an individual source has width. At a next higher level of organization

${ }^{1}$ All subsequent references to Rumsey relate to [15]. and grouping, an ensemble of sources has ensemble width. Such a grouping depends on the particulars of the scene and, for example, might variously be composed of the violin section, the string section or the whole orchestra. At the next highest level of organization for recorded music we can speak of room width and beyond that the width of the entire auditory scene. This scenebased approach isolates the 'what' from the 'where' and removes the conceptual confusion inherent in considering spatial attributes without addressing auditory grouping.

We can now propose spatial terminology to be applied to the relevant nested levels of auditory organization. Rumsey proposes that all spatial attributes be limited to five, three 'dimensional' attributes and two 'immersive' attributes. His three dimensional attributes--width, distance and depth---can be instanced by an individual sound source, an ensemble, room or scene (although it is unlikely that an individual sound source

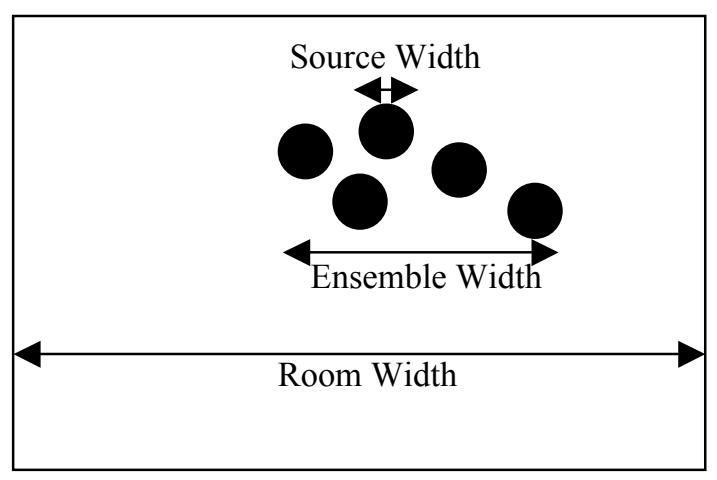

Scene

Figure 1. Spatial attributes of nested levels within an auditory scene (after Rumsey [15]).

could instance depth). Rumsey mentions that he is omitting height, we assume because his focus is on reproduction systems without elevation, but he also omits any other aspect of direction (even though he has contributed to its discussion in other research $[11,16]$ ). We will add direction to our list of spatial attributes ${ }^{2}$. Rumsey's category of immersive spatial attributes covers the domain of auditory spatial perception described by such interrelated terms as spatial impression, spaciousness and listener envelopment. Within this framework of scene analysis, Rumsey recommends the adoption of only two terms: envelopment and presence. He proposes that the term 'envelopment' be applied to the experience of being surrounded by sound either both from multiple sound sources and from a diffuse field such as reverberation. These are called 'source-related' and 'environmental' envelopment respectively. Rumsey has newly proposed that the term 'presence' be applied to the "sense of being inside of an (enclosed) space." We will adopt all of Rumsey's terminology (with our small addition) for consistency's sake and defer judgment on the use of the term 'presence' to future literature. Our resulting terminology can be summarized as follows:

\footnotetext{
${ }^{2}$ We will treat direction as a single, unambiguous perceptual attribute even though its geometrical representation requires two dimensions. The dominant role of the left-right axis in the play of perceptual grouping is considered later on.
} 
dimensional attributes:

width (individual/ensemble/room/scene)

distance (individual/ensemble/room/scene)

depth (individual in special cases/ensemble/ room/scene)

direction (individual/ensemble/room/scene)

immersive attributes:

envelopment (environmental/source-related) presence

Every level of organization within the scene can be characterized by the dimensional attributes, but only certain situations give rise to envelopment and presence.

\section{ELECTROACOUSTIC MUSIC}

\subsection{Spatial Attributes, Scene Analysis and Auditory Spatial Schemata}

Up to this point we have described a nested organization for scene analysis that is based in acoustic music. Rumsey's framework of source/ensemble/room/scene is intended for the subjective evaluation of the audio reproduction of music recordings. And, while the content of electroacoustic music can be quite dissimilar, the notion of nested levels of organization from micro to macro would appear to be quite valid for almost every kind of music. Rumsey's focus on typical audio content though complicates his framework in a way that inadvertently undermines the notion of a simple microto-macro organization. The shift from 'ensemble' to 'room' is not just a shift to the next higher level of nesting; it is a categorical shift that is normative only in the domain of acoustic music where instruments are indeed enclosed in rooms.

There are two issues revealed when considering the concept of 'room' in the context of electroacoustic music. The first issue is that the 'room' or the simulation of the 'room' can be accomplished by the treatment of an acoustic signal with a reverberator. Since the output of the reverberator is essentially a dense ensemble of duplicated signals, we have to ask why the 'room' is not considered a type of 'ensemble', essentially just another level of nesting in the organization? Then too, in the case that the original source signal is omitted, the reverberator's output itself essentially becomes a potential source. What then is the essential difference between a source, an ensemble and a room? The answer comes in response to the second issue. A 'room' is something different from a type of acoustic signal or treatment of a signal: it is an understanding that the listener constructs in response to auditory experience. 'Room' is part of our cognitive understanding formed in relation to auditory spatial schemata. Depending on the circumstances, 'room' may or may not be invoked by the listener in the process of forming an understanding of the auditory scene. In some circumstances we can imagine that 'room' is automatic and akin to schema-based stream segregation [3]. In other circumstances 'room' might well be an inference based on incomplete or fragmentary acoustic information. It is not the perceived nested relationships that determine the categories.
In response to the concept of 'room' presented by Rumsey [15], we have disentangled a confluence of terminology created by mixing the immediate and tangible spatial organization with patterns of spatial understanding. First, we have recognized that 'room' is not inherently a level in nested spatial relationships. Second, we have connected 'room' to the listener's cognitive spatial understanding and auditory spatial schemata. The original sense of the distinctions between source, ensemble and room has faded away. ('Source' and 'ensemble' are also discussed below in relation to auditory spatial schemata.) So, what remains of the original concept of a nested organization of spatial relationships? Clearly, the listener's perception of nested spatial relationships depends on the tangible circumstances, the particulars of the auditory scene. For electroacoustic music the elements in the nested structure are not limited a priori to one set of relational categories. At times, there may be no clear boundary between source and ensemble, that is, between one and many sources. A full description of spatial content and its implications within an artistic context may be very complex. Auditory spatial schemata may be stretched or even violated. Our ability to describe and categorize these complex interactions depends on having clear concepts and terminology so that we can recognize the crisscrossing of boundaries and the disruption of spatial norms in the artistic interplay.

\subsection{Spatial Schemata and Audio Reproduction}

Auditory spatial schemata are the recurrent patterns by which we understand the behavior of sound in space. All of our sensory capacities contribute to forming our core spatial schemata [7] and, therefore, auditory spatial schemata can be largely understood as projections of multimodal spatial schemata within the auditory domain. The listener makes sense of spatial sound first and foremost in terms of spatial schemata that are learned and reinforced in everyday life. The general schema of OBJECT gives rise to the auditory schema of SOURCE. The general schema of COLLECTION gives rise to ENSEMBLE. Both possess spatial attributes and typical spatial behaviors.

Sound localization is generally recognized as having a weak influence on the auditory scene. For that reason, spatial schemata have a particularly important role in spatial hearing because the schemata give coherence to spatial information that may otherwise be faint or incomplete. Spatial schemata are particularly important for audio reproduction when no other sensory information may collaborate the auditory spatial content. Our spatial schema for PATH gives coherence to motion effects that can otherwise be quite fragile. The listener makes sense of spatial relationships and creates a spatial organization. Then too, the disembodied sound of audio reproduction is often interpreted in a framework that is specific to this context. For example, the spatial arrangement of sources in a typical stereo pop song makes no physical sense. We accept the spatial arrangement as an idiom of audio reproduction, a musical-spatial idiom. The immaterial nature of audio reproduction enables auditory spatial art to exploit the spatial schemata of everyday life. 


\subsection{Play with Perceptual Grouping}

In electroacoustic music there is often a play of perceptual grouping that affects both the identification of sources and their spatial attributes. This play is typically driven by disruption at the level of perceptual event formation, disruption that can affect both grouping and localization mechanisms. There are numerous techniques that create such disruptions, techniques that typically can be manipulated to adjust the degree of disruption and thus enable the exploration of perceptual boundaries. While they may be conceptually different, these techniques often create similar results. In order to adequately describe the changes in the spatial attributes associated with these techniques, we need to distinguish four different frames of reference associated with the word 'source.' First there is the 'source signal,' that is the acoustic signal or a representation of the acoustic signal. Second, there is the tangible 'source image,' the 'source' that has spatial attributes in the auditory scene. There is the 'conceptual source,' the object that the listener identifies with the sound independent of its spatial attributes. ('Conceptual source' is related to Smalley's concept of source bonding [19].) Lastly, there is the listener's spatial schema, 'SOURCE.' The source image, whether it is understood as one or many conceptual sources, most likely is segregated as an auditory stream, and how the artistic play reshapes the listener's perceptual organization is discussed below.

Let us first consider the class of techniques that disrupt the identity of the source signal by breaking it into parts. These parts might be separated on the basis of time or spectrum (or both). A primary example of a temporal technique is granular processing that disrupts the temporal order of the acoustic source signal. A primary example of a spectral technique would be phase vocoding that alters the spectral organization of the acoustic source signal. Both of these techniques involve breaking the acoustic source signal into multiple parts, manipulating those parts and then assembling a result. (Of course, there are even more ways of using these tools.) The result can range from exact reconstruction of the acoustic source signal to the construction of something essentially new. The listener's conceptual source can range from the original conceptual source to a new source to multiple new sources. Also importantly, in both cases there is the possibility of assembling the result in one or more spatial locations.

Consider the two-dimensional field of possibilities for combinations of the width of the source image and the number of conceptual sources created by play with perceptual grouping (Figure 2). In the case that the acoustic source signal is perfectly reconstructed and that it is positioned in one spatial location, there is one conceptual source and one corresponding perceptual source. This matches the characteristics of SOURCE. In the case that manipulation of the source signal gives rise to multiple conceptual sources that are positioned in one spatial location, the result is multiple conceptual sources associated as one source image. (If there is a blurring between one or more conceptual sources, it may give rise to a blurring of whether there are one or more source images with the same spatial attributes.) In another case, if there are multiple conceptual sources and the component parts are spatially dispersed, then the spatial distribution tends to support a multiplicity of source images organized as an ENSEMBLE. An example is a spatially dispersed granular cloud [21,9].

Most interestingly, in the case that the identity of the source signal is maintained as one conceptual source but its component parts are spatially dispersed, there is a clear competition of perceptual organizations. The degree to which the spatialized component parts segregate from the whole (and border on multiple source images) determines a wide range of combined spatial/source percepts. At one end of the continuum is the possibility of a source image with an increased spatial width. At the other end is the possibility of multiple source images associated with one conceptual source (something that cognitive organization may fight). In between are the blurred boundaries of source/ensemble and of one/many source images. One particular example is the case when frequency bands are systematically spread in space giving rise to the perception of a single/multiple, source/ensemble distributed in space [20,9]. Granular synthesis and phase vocoding can be manipulated to create possibilities across the entire range of source image and conceptual source possibilities.

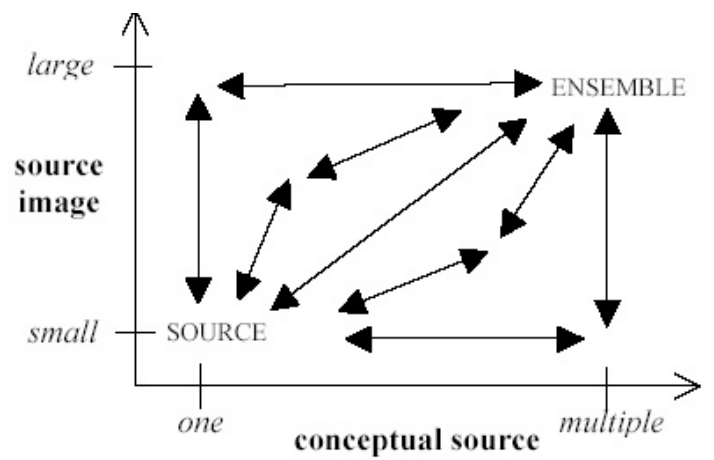

Figure 2. Two-dimensional field of possibilities for source image and conceptual source combinations created by play with perceptual grouping.

There is another class of techniques that often gives rise to similar boundary play. This second type of technique involves disruption of perceptual event formation by the manipulation of binaural information and is therefore a technique that primarily affects spatial attributes along the left-right lateral axis. A well-known recording technique provides a simple example. Imagine that two virtually identical vocal performances are recorded. One recording differs from the other only by micro-variations. If one recording is panned to the left loudspeaker and the other is panned to the right, the result is the perception of two instances of the same recording, one on the left and one on the right. The difference in micro-variations defeats the formation of a single spatial image. This is an extreme instance in which there is one conceptual source and two source images. More typically the differences between the ear signals causes the source image to increase in width. (The extent of the width can be related directly to the measured similarity between the signals arriving at the ears, often expressed as the interaural cross-correlation coefficient.) There are numerous ways of creating the micro-variations that give rise to binaural differences 
and every one of these can be achieved either by processing a source signal to create multiple versions or by synthesizing multiple source signals directly. The way in which the multiple source signals are created is immaterial. Creating small static frequency differences or static phase differences among source signals tends to give rise to a single conceptual source and a broadened source image [8]. Dynamic frequency differences (such as jitter or vibrato) provide a particularly effective way to manipulate the relationship between conceptual sources and source images. In the case that multiple source signals with dynamic frequency differences are gathered in one location (and do not create on-going binaural differences), the magnitude of frequency differences will position the conceptual source along a continuum from one to many while maintaining a single source image. A conventional example of this is singlechannel chorusing. In the case that the multiple source signals are spatially dispersed (in such a way as to create dynamic binaural differences at the listener's ears), then there will be a widened source image with one or more conceptual images. In the case that the dynamic frequency differences are of sufficient magnitude, then the broadened source image will break up into multiple images. But, of course, the power of vibrato to affect auditory grouping is well known from the demonstration by Steve McAdams with Roger Reynolds [13,14].

Up to this point we have discussed the impact of these two categories of processing techniques primarily on the spatial attribute of width, although other spatial attributes such as distance and depth are also often affected. When Rumsey asks the question of when source width becomes so wide as to create envelopment, he is pointing to a link between dimensional and immersive spatial attributes. When creating sound images that surround the listener, techniques of the first type tend to create 'source-related envelopment' while techniques of the second type tend to create 'environmental envelopment.' Techniques of the second type are closely related to artificial reverberation and the conditions under which the listener experiences envelopment in a reverberant field [10]. In fact, we can view multi-channel reverberation as yet another processing technique of the second type that produces a narrow to wide source image with a single conceptual source. Figure 3 illustrates how the results of reverberation and chorusing can be illustrated on the two-dimensional field of possibilities.

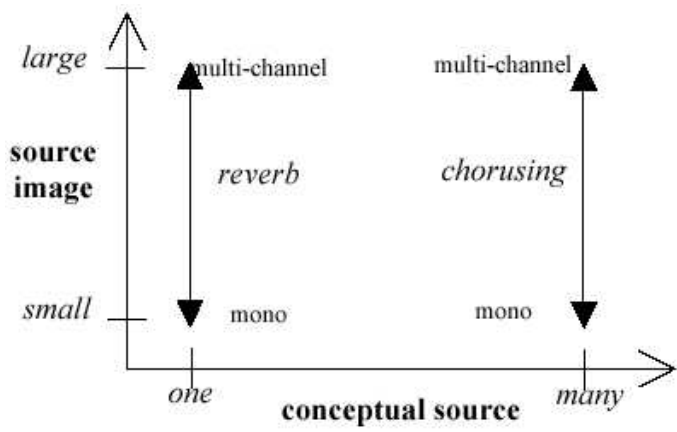

Figure 3. The results of reverberation and chorusing are considered on the two-dimensional field of possibilities.
While dynamic changes in spatial attributes have not been directly addressed here, it should be obvious that the techniques discussed can be implemented in a dynamic fashion. The two-dimensional field of possibilities can be transversed in a dynamic way. The experience of gradual change affecting auditory grouping and spatial organization is an aspect of numerous electroacoustic works, especially works of soundscape composition.

\subsection{Play with Auditory Spatial Schemata}

A good example of a general spatial schema with an auditory instantiation is CONTAINMENT. We construct our understanding of containment through an interaction with the world that involves all of our senses. That process includes the experience of objects moving into and out of other objects with their own internal space, a space that can contain another object. What we learn about CONTAINMENT as an auditory schema is that an object with internal space containing a sounding object transforms that sounding object depending on the characteristics of the container. Furthermore, a room is a kind of container, a particular type of container that can contain the listener as well as sounding objects. A room transforms sound in a different way than other containers, in part, because it also produces a sense of immersion for the listener when the listener is inside the room. These concepts are represented graphically in Figure 4.

a)

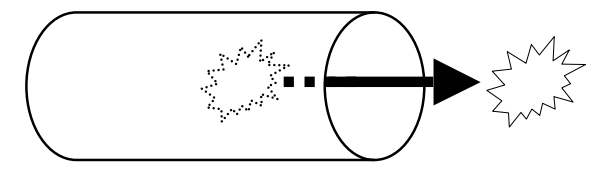

b)

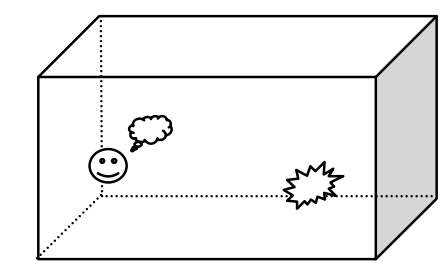

Figure 4. Auditory spatial schemata: a) representation of containment and $b$ ) representation of room.

Artistic play with auditory spatial schemata in electroacoustic music can occur in many ways. One way that is analogous to the previous examples is by manipulation of normal expectations. The violation of auditory spatial schemata has the effect of directing the listener's attention to content that is highlighted by the unusual or unexpected relationships. In this way the domain of the disruption becomes a subject for artistic expression. For example consider the possibility of the listener being inside of a container that is not a room. (The play of open space and contained space is a major element in Denis Smalley's Empty Vessels [17] where the listener's point of view is alternatively in an open space or inside of a large garden pot.) Consider also the possibility of the listener experiencing the sound of a source from one direction and the sound of the room containing the source coming from another. These possibilities are graphically represented in Figure 5. 
a)

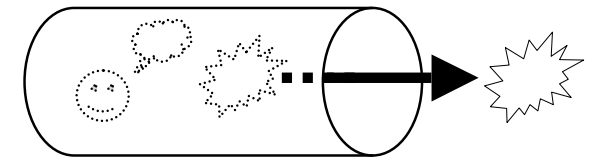

b)

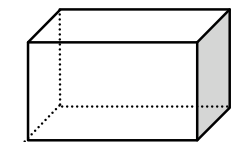

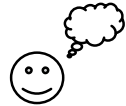

Figure 5. Violations of auditory spatial schemata: a) violation of containment and b) violation of room.

\section{CONCLUSION}

In his review of Bregman's book, Auditory Scene Analysis [4], David Huron says, "the act of hearing may be likened to the work of a cartographer constantly drafting maps of the auditory scene" [6]. Our discussion of spatiality in electroacoustic music has revealed some additional layers to that cartographer's job. Not only does the cartographer draft a diagram of the conceptual sources in the scene, but he/she also creates a spatial map of the source imagery. Spatial schemata aid the cartographer in bringing organization and understanding to the interrelationships between the conceptual sources and the spatial source imagery. And, although we have not touched on it here, the cartographer's knowledge of spaces and places experienced in the past provides a kind of mental atlas that provides additional levels of context and meaning. (Consider Denis Smalley's discussion of space in acousmatic music from this perspective [18]). And, while the cartographer might experience complete job satisfaction while dealing with the predictable spatial relationships of everyday life, he/she might also appreciate the peculiar charm experienced by the artistic play of spatial relationships presented in electroacoustic music.

\section{REFERENCES}

[1] Ando, Y. Architectural Acoustics: Blending Sound Sources, Sound Fields, and Listeners. SpringerVerlag, New York, 1998.

[2] Barron, M., and Marshall, A. H. "Spatial impression due to the early reflections in concert halls: The derivation of a physical measure," Journal of Sound. Vibration, 77(2), 1981.

[3] Bregman, A. S. Auditory Scene Analysis: The Perceptual Organization of sound. Cambridge. Massachusetts: The MIT Press, 1990.

[4] Griesinger, D. “The psychoacoustics of apparent source width, spaciousness and envelopment in performance spaces,' Acustica, 83, 1997.

[5] Harley, M. A. "Spatiality of sound and stream segregation in twentieth century instrumental music." Organised Sound 3(2), 1999.

[6] Huron, D. Book review: “Auditory Scene Analysis: The Perceptual Organization of Sound by Albert S. Bregman. Psychology of Music, 19(1), 1991.

[7] Johnson, M. The Body in the Mind: The Bodily Basis of Meaning, Imagination, and Reason. University of Chicago, 1987

[8] Kendall, G. "The Decorrelation of Audio Signals and Its Impact on Spatial Imagery." Computer Music Journal 19(4), 1995.

[9] Kim-Boyle, D. "Spectral and Granular Spatialization with Boids." SEAMUS 2007 National Conference, Ames, Iowa.

[10] Mason, R. Elicitation and measurement of auditory spatial attributes in reproduced sound. $\mathrm{PhD}$ Thesis, University of Surrey, 2002.

[11] Mason, R., Brookes, T., and Rumey, F. "Frequency dependency of the relationship between perceived auditory source width and the interaural crosscorrelation coefficient for time-invariant stimuli," $J$. Acoust. Soc. Am. 117(3), 2005.

[12] Mason, R., Brookes, T., and Rumey, F. “The effect of various source signal properties on measurements of the interaural crosscorrelation coefficient," Acoust. Sci. \& Tech. 26(2), 2005.

[13] McAdams, S. Spectral Fusion, Spectral Parsing, and the Formation of Auditory Images. Unpublished doctoral dissertation, Stanford University, 1984.

[14] McAdams, S. and E. Bigand. Thinking in Sound: The Cognitive Psychology of Human Audition. Oxford, England: Oxford University Press, 1993.

[15] Rumsey, F. "Spatial Quality Evaluation for reproduced Sound: Terminology, Meaning, and a Scene-based Paradigm." Journal of the AES, 50(9), 2002.

[16] Rumsey, F. "Subjective Evaluation of the Spatial Attributes of Reproduced Sound." Proceedings of the AES 15th International Conference, 1999.

[17] Smalley, D. Program notes to Empty Vessels included in the compact disk About Sources/scenes. IMED 0054, empreintes DIGITALes, 2000.

[18] Smalley, D. "Space-form and the acousmatic image." Organised Sound 12(1), 2007.

[19] Smalley, D. Spectromorphology and structuring processes. In S. Emmerson (ed.) The Language of Electroacoustic Music. Basingstoke: Macmillan Press, 1986.

[20] Torchia, R. and C. Lippe. "Techniques for MultiChannel Real-Time Spatial Distribution Using Frequency-Domain Processing." Proceedings of the 2004 Conference on New Interfaces for Musical Expression, Hamamatusu, Japan.

[21] Truax, B. "Composition and diffusion: space in sound in space." Organised Sound, 3(2), 1999.

[22] Zacharov, N. and K. Koivuniemi. "Unravelling the Perception of Spatial Sound Reproduction." Proceedings of the AES 19th International Conference, 2001. 